\title{
Association of Toxoplasma Gondii Infection with Spontaneous Abortion
}

\author{
MAHMUDA SULTANA ${ }^{1}$, MD.SAZZAD HOSSAIN², FARHANA DEWAN ${ }^{3}$, \\ JOBAIDA SULTANA ${ }^{4}$, MALIHA RASHID
}

\begin{abstract}
:
Objective: To evaluate whether there is any association of Toxoplasma gondii infection with spontaneous abortion.

Materials and Methods: This observational case control study was conducted in the Department of Obstetrics and Gynaecology, Dhaka Medical College Hospital, Dhaka (DMCH), over a period of one year from $31^{\text {st }}$ December 2008 to $30^{\text {th }}$ November 2009. A total 91 pregnant women were selected for this study. Among them 46 patients of spontaneous abortion were selected as case and 45 healthy pregnant women were selected as control purposively. Spontaneous abortion cases were diagnosed on the basis of history, clinical examination and USG findings. Patients with medical disorder, Rh-incompatibility, multiple pregnancy, cervical incompetence, septic abortion, threatened abortion, fibroid with pregnancy and uterine anomaly were excluded from the study. The clinical examination was done by standard method. Blood was collected from each subject, serum was separated. Main outcome measure was presence of toxoplasma antibodies in spontaneous abortion cases. Antibody was detected by ELISA method. A $p$ value of $<0.5$ was considered as significant.

Results: In both groups there was no difference in age, gestational age, socioeconomic status, living standard and educational status.

Toxoplasma specific IgM was detected in higher percentage of women with spontaneous abortion $(15.2 \%)$ than with controls (0\%), which is statistically significant $(<0.05)$. Seroprevalence for past Toxoplasma infection (IgG) was similar in both the groups, $17.4 \%$ and $8.9 \%$ for case and control respectively $(p>0.05)$. Though there is no association between seropositivity and level of education, place of living, contact with cat, occupational status, parity and past history of spontaneous abortion, seropositivity is higher in the poor socioeconomic group.
\end{abstract}

Conclusion: Seroprevalence for recent Toxoplasma gondii infection is higher in women with spontaneous abortion.

Key Words: Seroprevalence, Toxoplasma gondii , spontaneous abortion.

\section{Introduction:}

Toxoplasmosis is caused by the obligate intracellular parasite Toxoplasma Gondii ${ }^{1}$. The sexual form of the parasite lives in the gut of the definitive host, the cat, where it produces oocysts. After a period of maturing in the environment, these oocyts become the source of infection for secondary hosts, which may ingest them. In the secondary hosts (which include man,

1. Junior Consultant, Department of Obstetrics and Gynaecology, Shaheed Tajuddin Ahmed Medical College \& Hospital, Gazipur

2. Assistant Professor, Department of Medicine, Shaheed Tajuddin Ahmed Medical College \& Hospital, Gazipur

3. Professor \& Head, Department of Obstetrics and Gynaecology, Shaheed Suhrawardy Medical College \& Hospital, Dhaka

4. Assistant Professor, Department of Obstetrics and Gynaecology, Shaheed Suhrawardy Medical College \& Hospital, Dhaka

5. Professor of Obstetrics and Gynaecology,Former Head of, Department of Obstetrics and Gynaecology, Dhaka Medical College \& Hospital, Dhaka 
cattle, sheep, pigs, rodents and birds) there is disseminated infection. Following a successful immune response the infection is controlled, but dormant parasites remain encysted in host tissue for many years. The life cycle is completed when carnivorous felines eat infected animal tissue ${ }^{2}$.

It has been estimated that up-to one third of the world's population is infected by Toxoplasma gondii 3. In developed countries toxoplasmosis is the most common protozoal infection; around $22 \%$ of adults in UK are seropositive ${ }^{4}$. In countries like India or Brazil $42-60 \%$ of pregnant female are seropositive for toxoplasma 4 .

Toxoplasmosis can be transmitted to humans by three principal routes. It is estimated that approximately $50 \%$ of the toxoplasmosis cases in the United States are caused by eating inadequately cooked meat containing tissue cyst form of the organism ${ }^{5}$.

A second means of acquiring toxoplasmosis is by inadvertently ingesting the oocysts that cats have passed in their feces, either from a cat litter box, from infected soil where a cat has defecated or from unwashed fruits or vegetables that have contracted infected soil. A third means of transmission is transplacentally, when a primary infection occurs during pregnancy.

Maternal risks of toxoplasmosis in the immunocompetent gravida are minimal and $90 \%$ of infections are asymptomatic and self limited. Symptoms most commonly resemble infectious mononucleosis with posterior cervical or axillary lymphadenopathy that is discrete, nontender and nonsuppurtive. Other maternal symptoms may include malaise and muscle pain. Fetal risks, on the other hand, vary widely, depending on the trimester of acquisition and presence or absence of maternal treatment. In general the earlier the infection is acquired, the lower the risk of fetal transmission, but the greater the risk of serious sequelae to the fetus when congenital infection does develop ${ }^{6}$.

Maternal seroconversion occurring at or before five weeks gestation has been reported to have minimal to no risk of fetal infection, where as seroconversion in the third trimester may result in congenital infection in as many as $60 \%$ of neonates ${ }^{7}$. As many as $85 \%$ of congenitally infected neonates have subclinical infections that will later surface as chorioretinitis, hearing loss or developmental delays. Serious sequelae noted at or after birth, including intrauterine death, neurologic abnormalities, hydrocephalus, cerebral calcification, and chorioretinal scars with or without severe visual impairment, which may be reduced by the use of prenatal antibiotic therapy even though the rate of congenital infection may not change ${ }^{8}$.

Spontaneous abortion is the most common complication of pregnancy and is defined as the passing of a pregnancy prior to completion of the $20^{\text {th }}$ gestational weeks. It implies delivery of all or any part of the products of conception, with or without a fetus weighting less than $500 \mathrm{gm}$. Although the true incidence of spontaneous abortion is unknown, approximately $15 \%$ of clinically evident pregnancies and $60 \%$ of chemically evident pregnancies end in spontaneous abortion ${ }^{9}$.

An abnormal karyotype is the leading cause of spontaneous abortion. Other suspected causes of spontaneous abortion include infection, anatomic defects, endocrine factors, immunologic factors and maternal systemic illness. In a significant percentage of spontaneous abortion, the etiology is unknown ${ }^{9}$.

Primary toxoplasma infection acquiring during pregnancy may result in severe damage to the fetus including spontaneous abortion, IUGR, still birth, early neonatal death or congenital toxoplasmosis. Data indicate, however, that although chronic toxoplasmosis is associated with abortion it is not a common event 10. Silveria et al recently reported a case of maternofetal transmission in a preconceptionally immunized woman ${ }^{11}$. This finding could be accounted for by a down regulation of the T-cell mediated immune response that is observed during pregnancy ${ }^{12}$.

Acute and latent toxoplasma gondii infections during pregnancy are mostly diagnosed by serological test including detection of antitoxoplasma gondii specific IgM and IgG antibodies. Antibody screening programs aimed at the diagnosis of T.gondii infection among pregnant women have therefore been introduced in several countries ${ }^{13}$. In European countries, prevalence of toxoplasma gondii infection in pregnant woman varies from $9-67 \%{ }^{14}$. While prevalence as high as 
41.8 to $53.4 \%$ in pregnant women have been reported in Indian, Malaysian and Nepalese population ${ }^{14}$. In France and Austria, where the incidence of toxoplasmosis is much higher than in the United States, universal screening is mandatory ${ }^{15}$. In the United States, although universal screening has been frequently discussed, it is not generally practiced. Current evidence does not support routine screening for toxoplasmosis in UK ${ }^{16}$.

Toxoplasma infection is assumed to be one of the causes of spontaneous abortion. In Bangladesh the research on toxoplasmosis is a few though it is high risk area due to prevalence of domestic cat. Therefore, this case control study has been designed to investigate toxoplasma antibodies in a group of women with spontaneous abortion versus normal pregnancies and to assess whether there is an association between toxoplasmosis and spontaneous abortion.

\section{Materials and Methods:}

This observational case control study was conducted in the Department of Obstetrics and Gynaecology, Dhaka Medical College Hospital, Dhaka (DMCH), over a period of one year from $31^{\text {st }}$ December 2008 to $30^{\text {th }}$ November 2009. A total 91 pregnant women were selected for this study. Among them 46 patient of spontaneous abortion were selected as case and 45 healthy pregnant women were selected as control purposively.

A detailed history was taken from the patients including age of patients, gestational age, occupation, educational level, husband's and patient's monthly income, previous history of abortion, previous history of delivery of congenitally abnormal baby, complication during present pregnancy, history of medical and gynaecological disorders, previously done investigations etc. Socioeconomic status was classified by occupation, monthly income and educational level. A complete general and obstetric examination was done by per abdominal and per vaginal examination. Spontaneous abortion cases were diagnosed by history, clinical examination and USG findings. Normal healthy pregnant women were taken as control. All the controls were observed up to 20 weeks for any evidence of abortion. Informed written consent was taken from both the groups.
With usual aseptic precaution $5 \mathrm{ml}$ of venous blood was collected from each subject from the antecubital vein using disposable plastic syringe. Blood sample from spontaneous abortion cases was drawn immediately after or within 7 days of abortion. Serum was separated by centrifugation for 5 minutes at 3000 rpm at room temperature immediately after the blood was allowed to clot for 30 minutes. Separated serum was allocated in different eppendrop and preserved immediately at $-20^{\circ} \mathrm{C}$ temperature for subsequent analysis.

Anti-Toxoplasma IgG and IgM antibodies were investigated in the department of Microbiology,

The qualitative immunoenzymatic determination of IgM class antibodies against Toxoplasma gondii was based on the ELISA (Enzyme Linked Immunosorbent Assay) technique. The Toxoplasma IgM ELISA is an IgMm - capture ELISA.

The quantitative immunoenzymatic determination of IgG - class antibodies against T. gondii was also done by ELISA technique.

If only IgM or both IgM and IgG are positive, then the person was considered as infected within past 6 months. If only lgG is positive it is considered that the person is infected at least 6 months before.

Patients with medical disorder, Rh-incompatibility, multiple pregnancy, cervical incompetence, septic abortion, threatened abortion, fibroid with pregnancy and uterine anomaly were excluded from the study. Main outcome measure was presence of toxoplasma antibodies in spontaneous abortion cases. Ethical clearance and permission for the study was taken from appropriate authority.

Data were processed and analyzed using SPSS version 16.0. Chi-square $\left(x^{2}\right)$ test, unpaired students t-test, and Fisher's exact test were done for test of significance where appropriate. A p value of $<0.05$ was considered as significant.

\section{Results:}

The findings of the present study shows the fact that the case and control populations were similar in age, socioeconomic status, educational status, place of living, occupational status, gestational age and without any significant difference between the groups. 
Table-I

Characteristics of the patients

\begin{tabular}{|c|c|c|c|c|c|}
\hline Characteristics & & & & & Significance \\
\hline & & & & & \\
\hline Age (Yrs) & & & & & 0.830 \\
\hline Gestational age - GA (Weeks) & & & & & 0.997 \\
\hline & $\mathrm{N}$ & $\%$ & $\mathrm{~N}$ & $\%$ & \\
\hline Socioeconomic status & & & & & \\
\hline Upper class & 2 & 4.3 & 1 & 2.2 & \\
\hline Middle class & 28 & 60.9 & 29 & 64.4 & 0.830 \\
\hline Poor & 16 & 34.8 & 15 & 33.3 & \\
\hline Place of living & & & & & \\
\hline Urban & 19 & 41.3 & 20 & 44.4 & \\
\hline Semi-urban & 13 & 28.3 & 13 & 28.9 & 0.919 \\
\hline Rural & 14 & 30.4 & 12 & 26.7 & \\
\hline Gravida & & & & & \\
\hline Primi & 14 & 30.4 & 13 & 28.9 & 0.871 \\
\hline Multi & 32 & 69.6 & 32 & 71.1 & \\
\hline Contact with cat & & & & & \\
\hline Present & 41 & 89.1 & 42 & 93.3 & 0.369 \\
\hline Absent & 5 & 10.9 & 3 & 6.7 & \\
\hline
\end{tabular}

Table-II

Presence of immunoglobulin in serum

\begin{tabular}{lcccccc}
\hline Immunoglobulin & \multicolumn{2}{c}{ Case (46) } & & \multicolumn{2}{c}{ Control (45) } & Significance \\
\cline { 2 - 3 } & $\mathrm{N}$ & $\%$ & & $\mathrm{~N}$ & $\%$ & \\
\hline $\lg \mathrm{M}$ & 7 & 15.2 & & 0 & 00 & 0.007 \\
$\quad$ Positive & 39 & 84.8 & & 45 & 100 & \\
$\quad$ Negative & 8 & 17.4 & & 4 & 8.9 & 0.231 \\
$\lg$ Positive & 38 & 82.6 & 41 & 91.1 & \\
$\quad$ Negative & & &
\end{tabular}

Table III

Distribution of the study subjects by Seropositivity, either IgG or IgM

\begin{tabular}{lcccc}
\hline Characteristics & No of women (91) & \multicolumn{2}{c}{ Seropositive } & P value \\
\cline { 3 - 4 } & & $\mathrm{N}$ & $\%$ & \\
\hline Contact with cat & & & & \\
$\quad$ Present & 83 & 14 & 16.9 & 0.751 \\
$\quad$ Absent & 8 & 1 & 12.5 & \\
Gravidity & 27 & 9 & 22.2 & \\
$\quad$ Primi & 64 & 14.1 & 0.338 \\
$\quad$ Multi & 3 & 0 & 00 & \\
Previous congenital anomaly & 88 & 15 & 17 & 0.578 \\
$\quad$ Present & 3 & 1 & 33.3 & \\
$\quad$ Absent & 57 & 5 & 8.8 & 0.036 \\
Social Class & 31 & 9 & 29.0 & \\
$\quad$ Upper & & & \\
$\quad$ Middle & 39 & 5 & 12.8 & \\
$\quad$ Poor & 26 & 2 & 7.7 & 0.058 \\
Place of living & 26 & 8 & 30.8 & \\
$\quad$ Urban & & & & \\
$\quad$ Semi urban & Rural & & & \\
\hline
\end{tabular}

Result shown by $\chi^{2}$ test 
Table-IX

IgG status among the past history of spontaneous abortion of the study subjects $(n=21)$

\begin{tabular}{lcccccc}
\hline IgG & \multicolumn{2}{c}{ Case (10) } & & \multicolumn{2}{c}{ Control (11) } & Pvalue \\
\cline { 2 - 3 } \cline { 5 - 7 } & $\mathrm{N}$ & $\%$ & & $\mathrm{~N}$ & $\%$ & \\
\hline Positive & 1 & 10.00 & & 2 & 18.2 & .537 \\
Negative & 9 & 90.0 & & 9 & 81.8 & \\
\hline
\end{tabular}

Table I shows the demographic characteristics of the patient and shows no difference between the groups (>0.05).

Table II shows that IgM was positive in $7(15.2 \%)$ patients in case group and none in control group and the difference was statistically significant $(p<0.05)$. Whereas 8 (17.4\%) and 4 (8.9\%) patients had IgG positive in case and control group respectively, which shows no significant difference ( $p>0.05)$.

There is no relation of seropositivity with contact with cat, number of pregnancy and history of previous pregnancy. But significant association is found with social class and place of living $(p<0.05)$. Rural and lower socioeconomic people are more infected with toxoplasma gondii.

There is no relation with presence of IgG that is sign of previous infection with spontaneous abortion.

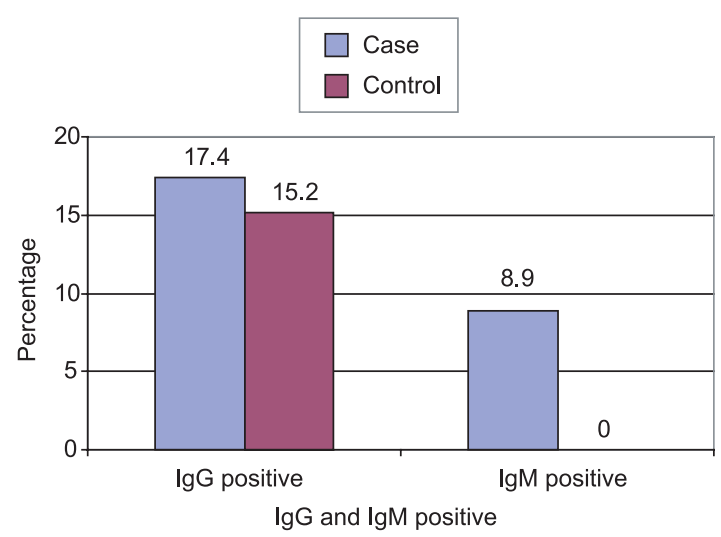

Fig 1: Bar diagram showing the IgG and IgM positive in case and control group ( $n=91)$

This diagram (Fig 1) shows the presence of recent (Ig $M$ ) and old (Ig G) infection in both groups. Sign of old infection present in both groups without any significant difference. On the other hand recent infection is found in $15.2 \%$ cases in spontaneous abortion group only. No IgM found in control group, which indicates that there is a strong association of spontaneous abortion and toxoplasma gondii infection.

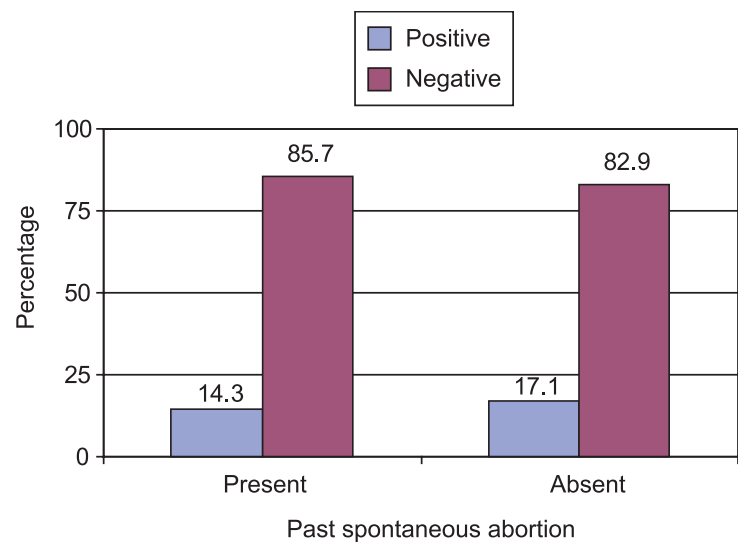

Fig 2: Bar diagram showing theseropositive status between past history of spontaneous abortion and no history of spontaneous abortion of the study subjects $(n=91)$

Figure 2 shows seropositive (either IgG or IgM) was found in $3(14.3 \%)$ patients who had past spontaneous abortion and in 12 (17.1\%) who had no past spontaneous abortion and the difference was not statistically significant $(p>0.05)$ between two groups. ( $P=0.756$, $p$ value reached from Chi-Square test).

\section{Discussion:}

Most acute toxoplasma infection are relatively asymptomatic, therefore, a universal challenge for obstetrician is how to identify acute infections during pregnancy. The method of choice in the diagnosis is by detection of specific antibodies in the patient's serum. Different studies have been done to see the association between toxoplasma gondii infection with spontaneous abortion. Many studies have demonstrated the relationship between toxoplasma gondii infection with spontaneous abortion ${ }^{17,18,19,20,21}$; while others have refuted an association ${ }^{22}$.

The purpose of present study was to see the seroprevalence of toxoplasma gondii infection in 
spontaneous abortion. A case control study with 46 cases of spontaneous abortion and 45 healthy pregnant women (control) was designed. The finding of the present study is strengthened by the fact that the case and control populations were similar in age, mostly were third decade. Socioeconomic status of both group were similar. Regarding obstetric variable like gestational age and gravidities were homogenously distributed without any significant difference between the groups.

The serological data of this study indicates that there was a clear association between acute toxoplasma infection and spontaneous abortion. IgM antibody was positive in $15.2 \%$ of cases and $0 \%$ of controls $(p<0.05)$ of our series. Strong association of presence of IgM antibody and spontaneous abortion also was found in $10.52 \%{ }^{19}, 34.5 \%{ }^{18}$ and $23.3 \%{ }^{20}$ cases in different studies. Although other one study shows only $0.97 \%$ IgM positive in spontaneous abortion ${ }^{23}$ majority shows strong association of IgM antibodies and spontaneous abortions ${ }^{18-20 .}$

Regarding the IgG status, $17.4 \%$ of cases and $8.9 \%$ controls were IgG positive. But the difference is not statistically significant $(p=>0.05)$. But a study shows significantly higher seroprevalence of toxoplasmosis in habitual abortion ${ }^{24}$.

In this study there is no significant difference in seropositivity (IgG) between patient with past history of spontaneous abortion and patient with no such history $(p=>0.05)$. But study shows that seroprevalence rate is increased with a greater number of previous abortions ${ }^{24}$.

Most of the patients had IgG positive both in case and control groups but we did not find any association between presence of IgG and abortion, as rate was similar in both groups. It indicates that old infection is not responsible for abortion. There was no relation with seropositivity with age, gestational age, gravidity and previous history of congenital anomaly. Except age same findings showed by other two studies ${ }^{14,25}$ and one showed increasing seropositivity with age 25. Pet animals particularly cat is a major source of transmission of Toxoplasma infection in human and Muna et al showed higher seropositivity among the patients who had close contact with cat ${ }^{25}$. But we did not find any association between seropositivity and contact of cat.
Is there any relation with social class and living standard of women with Toxoplasma infection? As eating of improperly washed or unwashed fruits and vegetables those have contact with contaminated soil from cat's feces might responsible for transmission of infection. Living standard and social class might have some relation. Alvarado-Esquivel et al showed significantly higher seroprevalence ( $14 \%$ vs $6 \%$, $\mathrm{p}<0.05$ ) in women from lower social class than from higher social class ${ }^{25}$. Nash et al showed significantly higher seroprevalence in women from rural areas 26 . We also found higher seroprevalence in women from rural areas and from lower social class.

\section{Conclusion:}

This study has shown that seroprevalence for recent toxoplasma gondii infection is higher in women with spontaneous abortion. But this study failed to identify any association between latent toxoplasma gondii infection and spontaneous abortion.

\section{Referrences:}

1. Ernest JM, 'Parasitic infections' in James, Weiner Steer and Gonik (eds.) High risk pregnancy: management options. Elsevier, India, Third edition, 2006; pp.710-713.

2. Finch RG, Moss P, Jeffries DJ and Anderson J 'Infectious disease, tropical medicine and sexually transmitted diseases', . Kumar and Clark Clinical Medicine, $6^{\text {th }}$ edn, Elsevier New York. 2005.

3. Montoya JG, Liesenfeld O. 'Toxoplasmosis'. Lancet. 2004; 363: 1965-76.

4. Todd WT, Lock Wood DNJ, Sundar S . 'Infectious diseases'. In: Boon, NA, Colledge, NR, Walker, BR and hunter, JA (eds). Davidson's Principal and Practice of Medicine. 20 th edn. Elsevier, New York,2006.

5. Roghmam MC, Fulkner CT, Lefkowitz A. Decreased seroprevalence for toxoplasma gondii in seventh day Adventists in Maryland. Am J Trop Med. 1999; 60: 790-92.

6. Hohlfeld P, Daffos F, Tlaulliez P et al. Fetal Toxoplasmosis: outcome of pregnancy and infant follow up after in ultero treatment. J Pediatr.1989; 115: 765-69.

7. Forestier F, Daffos F, Hohlfeld P, Lynch L. Infectious fetal diseases prevention, prenatal 
diagnosis, practical matters. Presse Med.1991; 20:1448-54.

8. Foulon, W, Pinon JM, Stray-Pedersen B. Prenatal diagnosis of congenital toxoplasmosis: a multicenter evaluation of different diagnostic parameters. . Am J Obstet Gynecol,1999; 118: 843-48.

9. Peter S, Uzelac S, Garnel . 'Early pregnancy risk', In Decherney AH, Nathan, L, Goodwin, T, Laufer, N (eds.). Current Obstetrics \& Gynaecologic Diagnosis and Treatment, $10^{\text {th }}$ edn, McGraw-Hill, New York 2007; 259-66.

10. Arias F, Daffary SN \& Bhide AG . 'Fetal infections'Practical guide to high risk pregnancy and delivery. . $3^{\text {rd }}$ edn. Elsevier, New Delhi, India,2008; pp. 160-64.

11. Silveria C, Ferreira R, Muccioli C, Nussenblatt $\mathrm{R}$, Belfort R. Toxoplasmosis transmitted to a newborn from the mother infected 20 years earlier. Am J Ophthalmo.I 2003;136: 370-71.

12. Garweg JG, Scherrer J, Wallon M, Kodjikian L, Peyron F. Reactivation of ocular toxoplasmosis during pregnancy. BJOG 2005;112: 241-42.

13. Kumar A, Arora V, Mathur M. Toxoplasma antibody levels in females with habitual or sporadic abortions and normal pregnancies. Ind J Med Microbiol 2004; 22: 276-77.

14. Esquivel CA, Alvarez AS, Durate GS, Martinez SE . Seroepidemiology of toxoplasma gondii infection in pregnant women in a public hospital in northern Mexico. BMC infectious Diseases 2006;6: Viewed 13 July 2006, http:// www.biomedcentral.com/1471-2334/6/113

15. Jeannel D, Costagliola D, Niel G. What is known about the prevention of congenital toxoplasmosis? Lancet.1990; 336: 359-61

16. Overton TG . 'Antenatal care', in Edmonds DK (eds),. Dewhurt's Textbook of Obstetrics and Gynaecology, $7^{\text {th }}$ edn, Blackwell Publishing Oxford. 2007; 39-45.
17. Lolis D, Tzigounis V, Michalac S, Koumentakou, Kaskarelis D E. 'Int J Gynaecol Obstet.1978; 15(4): 299-301.

18. Zargar AH, Waani GR, Laway BA, Kakroo DP, Sof BA. Toxoplasmosis in women with recurrent abortions, neonatal death and its treatment outcome. Ind JPathol Microbiol.1999; 42( 4): 481-83.

19. Turbadkar D, Mathur M, Rele M. Seroprevalence of torch infection in bad obstetric history. Ind J Med Microbiol 2003; 21(2):108-10.

20. Yasodhara P, Rama Lakshi BA, Lakshmi V, Krisna TP. Socioeconomic status and prevalence of toxoplasmosis during pregnancy. Ind J Med Microbiol.2004; 22: 241-43.

21. Chen KT, Esklid A, Bresnahan M, Pedersen B, Sher A, Jenum PA. Previous maternal infection with toxoplasma gondii and the risk of fetal death. Am J Obstet Gynaecol 2005;193:443-49.

22. Djurkovic-Dijakovic O. Toxoplasma infection and pathologic outcome of pregnancy. Gynecol Obstet Inv.1995; 40( 1): 36-41.

23. Razzak $A H$, Wais $S A$, Saeid AY .Toxoplasmosis: the innocent suspect of pregnancy wastage in Duhok, Iraq. Eastern Medi Health J.2005; 11:625-32.

24. Muna M, Hamdani Al, and Mahdi NK. Toxoplasmosis among women with habitual abortion. Eastern Medi Health J.1997; 3: 310-15.

25. Alvarado-Esquivel C, Torru C, Liesenfeld O, Lopez CR, Martinez ES, Alvarezm S, 'Seroepidemiology in toxoplasma gondii infection in pregnant women in rural Durango, Mexico'. J Parasitol.2009;95(2):271-74.

26. Nash JQ, Chissel S, Jones J, Warburton F, Verlander NQ . Risk factors for toxoplasmosis in pregnant women in kent, United Kingdom. Cambridge J.2005;133: 475-33. 